SLAC-PUB-15853

LCLS-II-TN-13-02

December 2013

\title{
A Possible Dechirper Device for the LCLS and LCLS-II
}

\author{
K. Bane, P. Emma, Z. Huang, R. Iverson, \\ T. Raubenheimer, G. Stupakov, L. Wang (SLAC)
}




\section{INTRODUCTION}

The purpose of this note is to investigate the use of a metallic pipe with small corrugations [1] for passively dechirping, through its wakefield, the LCLS beam. Similar dechirper systems have been tested in Pohang and Brookhaven at relatively low energies $(<100 \mathrm{MeV})$ and with relatively long bunches $(>1$ ps) $[2,3]$. A recent proposal from RadiaBeam is to build a 2-m dechirper and install it in the LCLS for a GeV, femtosecond beam test. Such a device can be used to either reduce or increase the amount of energy chirp in a short electron bunch prior to the FEL interaction in the undulator, and hence provide a flexible control of the LCLS FEL bandwidth. The results can be used to guide the design of the LCLS-II dechirper, where it is important to remove unwanted electron energy chirp from the LCLS-II superconducting accelerator and to provide independent controls of x-ray properties in each of the two parallel beamlines.

Details of the longitudinal and transverse wake calculations in a flat, corrugated pipe, as well as Figs. 2-4 of this note, can be found in Ref. [4]. In this note in Fig. 1 we give a sketch of the dechirper in longitudinal view, showing the parameters half-gap $a$, corrugation period $p, t=p / 2$, and depth $h$. The beam and structure parameters that will be used in the example calculations of this note are given in Table I. Note that corrugation parameters in the table are at the moment initial suggested values only, and that the calculated wake effects are obtained using approximate analytical formulas. For deciding on the actual corrugation parameters of a dechirper to be built, one will need to perform numerical calculations of the wakes, by e.g. using the field matching

program described in Ref. [5]. Such calculations are not within the scope of this note. 


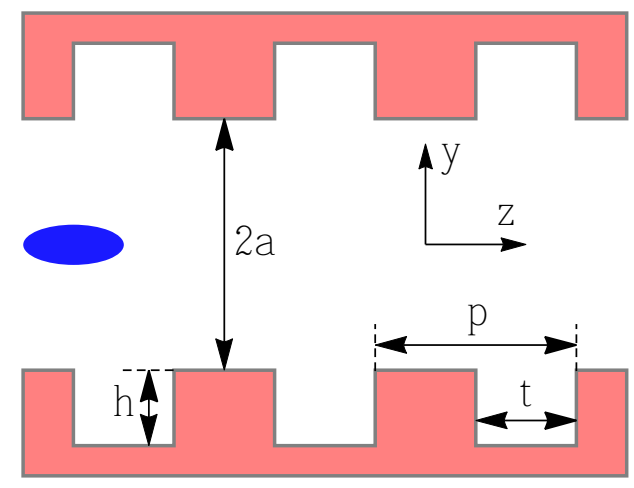

FIG. 1. Geometry in longitudinal view of the vacuum chamber with corrugated walls. A rectangular coordinate system is centered on the symmetry axis of the chamber. The blue ellipse represents an electron beam propagating along the $z$ axis.

TABLE I. Beam and structure parameters used in the example calculations in this note. Here the structure parameter $t$ is taken to be equal to $p / 2$.

\begin{tabular}{||l|c|c|c||}
\hline Parameter name & LCLS & LCLS-II & Unit \\
\hline \hline Beam energy, $E$ & 6.6 & 4.0 & $\mathrm{GeV}$ \\
Beam charge, $Q$ & 250 & 100 & $\mathrm{pC}$ \\
Peak current, $\hat{I}$ & 1.2 & 1.0 & $\mathrm{kA}$ \\
Structure: & & & \\
$\quad$ Length, $L$ & 2 & 10 & $\mathrm{~m}$ \\
$\quad$ Depth, $h$ & 0.25 & 0.5 & $\mathrm{~mm}$ \\
$\quad$ Period, $p$ & 0.25 & 1.0 & $\mathrm{~mm}$ \\
Example gap, $2 a$ & 1.6 & 4.0 & $\mathrm{~mm}$ \\
\hline \hline
\end{tabular}




\section{DECHIRPER DESIGN PARAMETERS AND EXPECTED EFFECTS ON THE BEAM}

The choice of dechirper parameters is determined from the following considerations. A smaller dechirper gap generates a stronger longitudinal wake (which scales as the inverse square of the gap), and allows one to shorten the device for the required size of energy chirp. However, an extremely small gap makes the transverse dipole and quadrupole wakes stronger (they scale as the inverse fourth power of the gap) and leads to projected emittance growth and tight tolerances on the beam offset in the dechirper.

In Fig. 2 we plot the energy difference between the tail and head of the bunch due to the dechirper as function of half-aperture $a$. This function depends on $a$ as $a^{-2}$. For the plot we assume that the charge $Q=250 \mathrm{pC}$ and structure length $L=1 \mathrm{~m}$. We see that for $a=0.7 \mathrm{~mm}, \Delta E=-10 \mathrm{MeV}$.

We illustrate the strength of the transverse wakes in Figs. 3 and 4. In Fig. 3 we plot the relative project emittance increase $\left(\epsilon / \epsilon_{0}\right)-1$ for a bunch traversing the structure on axis $v s$. half-aperture $a$. The emittance growth is due to the quad wake. The parameters used are ones that could apply to the LCLS: bunch charge $Q=250 \mathrm{pC}$, focusing function $\beta_{y}=10 \mathrm{~m}$, rms bunch length $\sigma_{z}=10 \mu \mathrm{m}$, length of structure $L=1 \mathrm{~m}$, and beam energy $E=6.6 \mathrm{GeV}$. We see that for these parameters, the half aperture of the dechirper needs to be kept to $a \gtrsim 0.6 \mathrm{~mm}$. As $a$ becomes smaller, the emittance grows steeply. However, if one made a dechirping structure composed of two identical parts, with the second half rotated by 90 degrees with respect to the first half, and with equal beta functions $\left(\beta_{x}=\beta_{y}\right)$ then the quad wake effect will cancel (assuming the beta function is large compared to the structure length).

If the beam enters the structure vertically offset from the axis by $y=y_{0}$, it will excite both dipole and quadrupole wakefields. Let us denote the jitter 


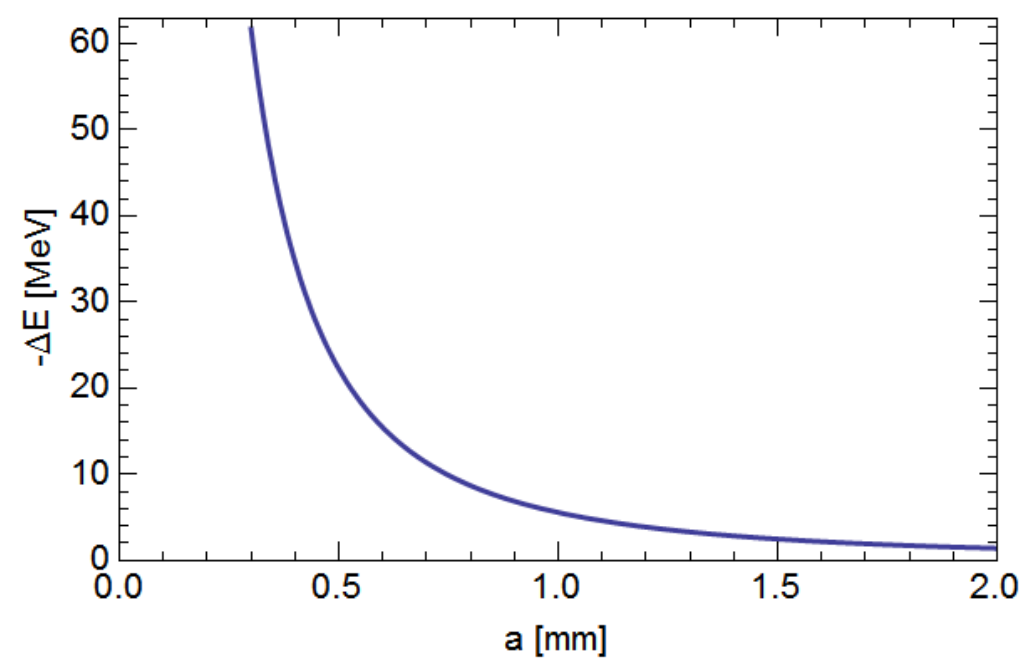

FIG. 2. Energy difference at tail of a short bunch compared to the head due to the wakefield of a flat dechirper vs. half-aperture $a$ [4]. Here charge $Q=250 \mathrm{pC}$ and structure length $L=1 \mathrm{~m}$.

amplification factor by the symbol $\xi$ (for details of the meaning of this factor, see Ref. [4]). The jitter amplification factor $\xi$ as function of half aperture $a$ is plotted in Fig. 4. Note that the other parameters are the same as those used in the earlier quad wake calculations. We see that one needs to keep the half aperture of the dechirper jaws to $a \gtrsim 0.7 \mathrm{~mm}$ in order to keep $\xi \lesssim 1.2$.

The results presented above indicate that the transverse wake limit the minimal gap $2 a$ between 1 and $2 \mathrm{~mm}$ (smaller beam charge would allow a somewhat smaller gap). Given that, in the typical setup, we would like the size of the corrugations to be a small fraction of the minimal gap, we suggest a flat corrugated pipe with these parameters: depth $(h)=\operatorname{period}(p)=0.25 \mathrm{~mm}$, $t=p / 2=0.125 \mathrm{~mm}$, and length of pipe $L=2 \mathrm{~m}$. The gap is tunable, with minimum full gap $2 a=1 \mathrm{~mm}$. It is desirable to split the 2-m-long dechirper 


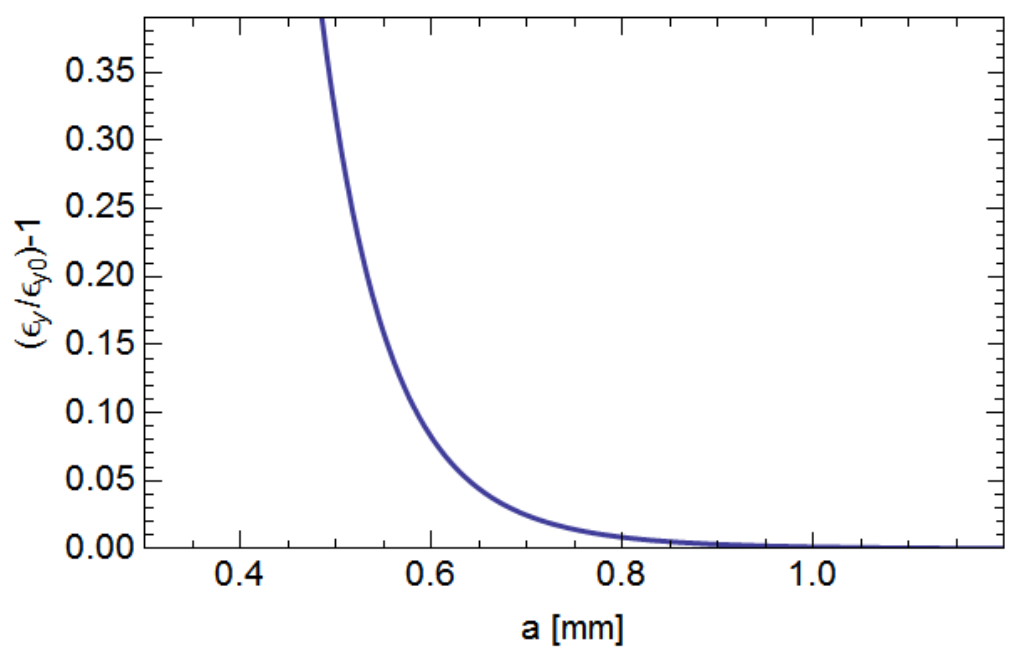

FIG. 3. Relative emittance growth due to the quad wake in a flat dechirper vs. halfaperture $a$ [4]. Here charge $Q=250 \mathrm{pC}$, energy $E=6.6 \mathrm{GeV}$, rms bunch length $\sigma_{z}=10 \mu \mathrm{m}$, and structure length $L=1 \mathrm{~m}$. The beam is on-axis; it has a longitudinal uniform distribution, and initially an uncorrelated, bigaussian transverse distribution in $y y^{\prime}$ phase space.

into two segments of $1 \mathrm{~m}$ length each, rotated by 90 degrees relative to each other. Note that the longitudinal wakefield generated by such a $2-\mathrm{m}$ device is already $25 \%$ stronger than the $553 \mathrm{~m}$ of Linac L3 in the LCLS.

\section{LCLS CONSIDERATIONS}

A possible location for the 2-m-long dechirper is the LCLS LTU area, just upstream of QDL34 (as close to XCDL4 as possible). XCDL4 is at $\mathrm{z}=3377.706$ in linac $\mathrm{z}$ coordinates. The beta functions are $\beta_{x}=5 \mathrm{~m}$, and $\beta_{y}=17 \mathrm{~m}$. It is conceivable to make $\beta_{x}=\beta_{y}$ there but this has not been studied carefully.

Using a linac longitudinal beam dynamics tracking code (LiTrack [6]), we 


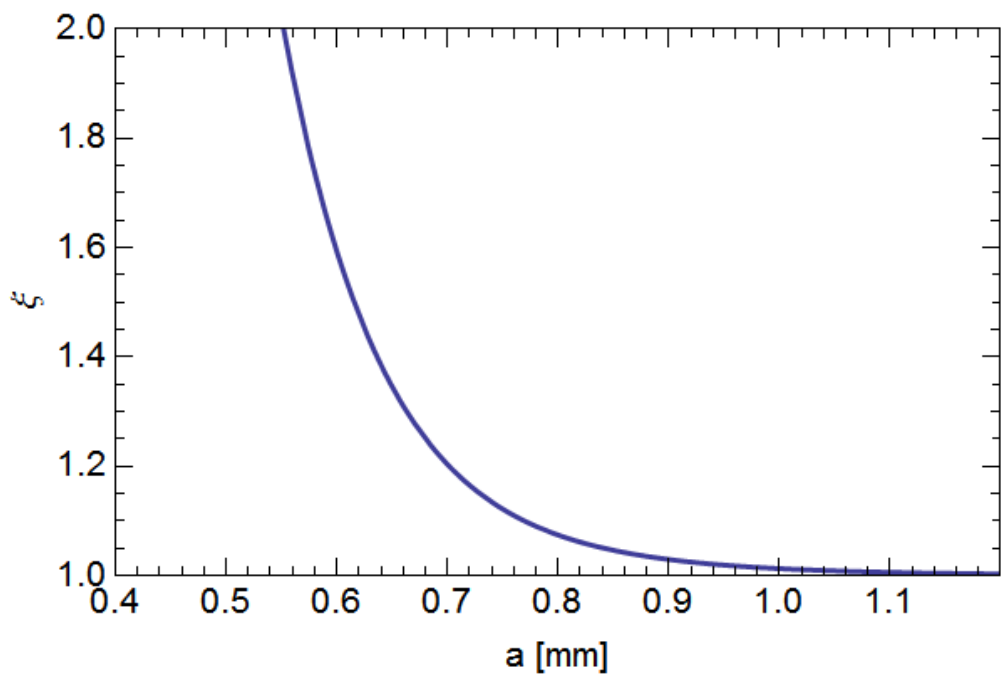

FIG. 4. Jitter amplification factor $\xi$ due to the dipole wake in a flat dechirper $v s$. half-aperture $a$ [4]. Here charge $Q=250 \mathrm{pC}$, energy $E=6.6 \mathrm{GeV}$, rms bunch length $\sigma_{z}=10 \mu \mathrm{m}$, and structure length $L=1 \mathrm{~m}$. The beam has a longitudinal uniform distribution, and initially an uncorrelated, bigaussian transverse distribution in $y y^{\prime}$ phase space.

give two examples of possible uses of a dechirper in the LCLS. We consider a 250 -pC beam that is accelerated to $6.6 \mathrm{GeV}$, in order to generate soft $\mathrm{x}$ rays at the $2 \mathrm{keV}$ photon energy. In Fig.5 (a) we show a possible outcome of longitudinal phase space at the end of L3. [Here and in following figures, we show a group of 3 or 4 plots giving: phase space (upper right), beam current (lower right), (sometimes) longitudinal wake of dechirper (lower left), and beam energy spread (upper left).] The longitudinal phase space has a residual chirp with a FWHM energy spread of $0.17 \%$, which means the FEL bandwidth will be $0.34 \%$ determined by the energy chirp. By setting the full gap of the dechirper to $2 a=1.58 \mathrm{~mm}$, the residual chirp can be removed to a 
large degree, as shown in Fig. 5 (b). The FEL bandwidth can be reduced to 0.1-0.2\% level, set by the intrinsic SASE bandwidth. The spectral brightness will be increased by a factor of 2 accordingly.

As another example, we consider producing large bandwidth FEL radiation for femtosecond x-ray nanocrystallography [7]. In such experiments, nanocrystals are randomly hit by XFEL pulses, producing diffraction patterns at unknown orientations. One can determine these orientations by studying the diffraction patterns themselves, i.e. by indexing the Bragg peaks. The number of indexed individual images and the SASE bandwidth are inherently linked, because increasing the number of Bragg peaks per individual image requires increasing the bandwidth of the spectrum. This calls for a few percent SASE bandwidth, resulting in an increase in the number of indexed images at the same number of hits.

Large bandwidth FEL has been generated by over-compressing electron bunches in $\mathrm{BC} 2$, and 1-2\% bandwidth (FWHM) has been observed using soft x-ray spectrometers [8]. In Fig. 6 (a), we show an example of an overcompressed 250-pC bunch at $6.6 \mathrm{GeV}$ (L3 end), the FWHM electron energy spread is $1.55 \%$. By closing the full gap of the dechirper to $1 \mathrm{~mm}$, this energy spread can be increased to 2.33\%, as shown in Fig. 6 (b). There could be emittance growth associated with this extreme gap and further studies are required to quantify this effect.

Note BPMDL4 is adjacent to the proposed dechirper device and existing downstream BPMs can be used to detect the beam kick from the de-chirper. Projected emittance, spectrum, XTCAV and FEL diagnostics are already in place in the LCLS. 

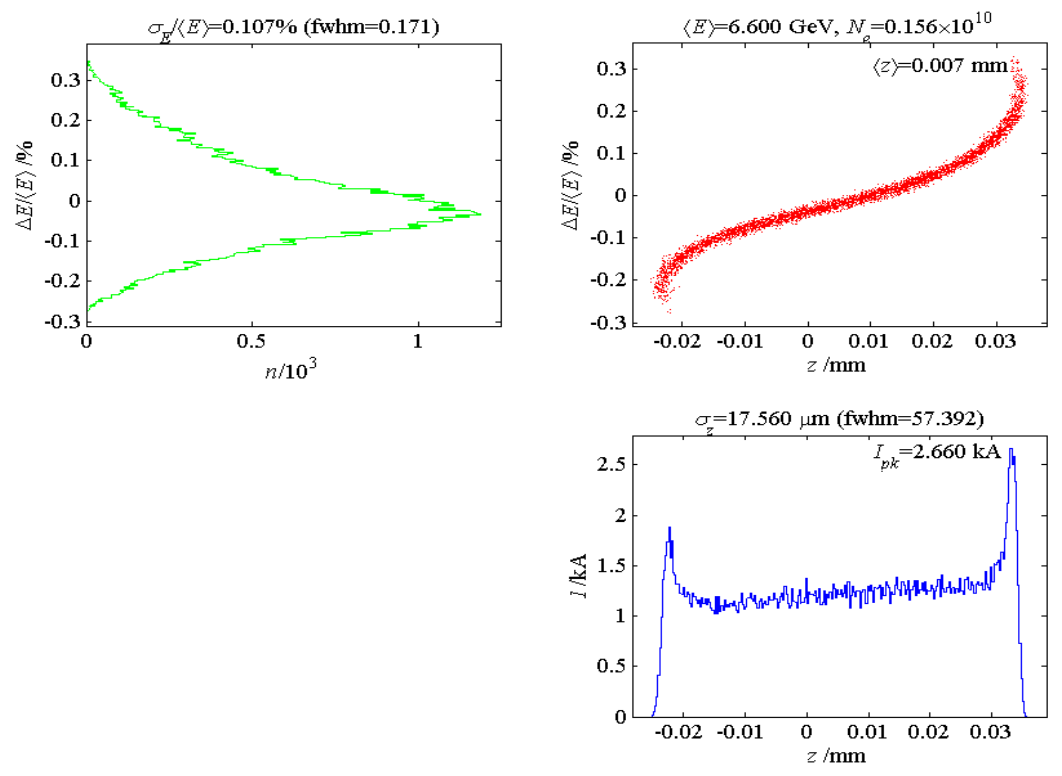

(a)Without dechirper.
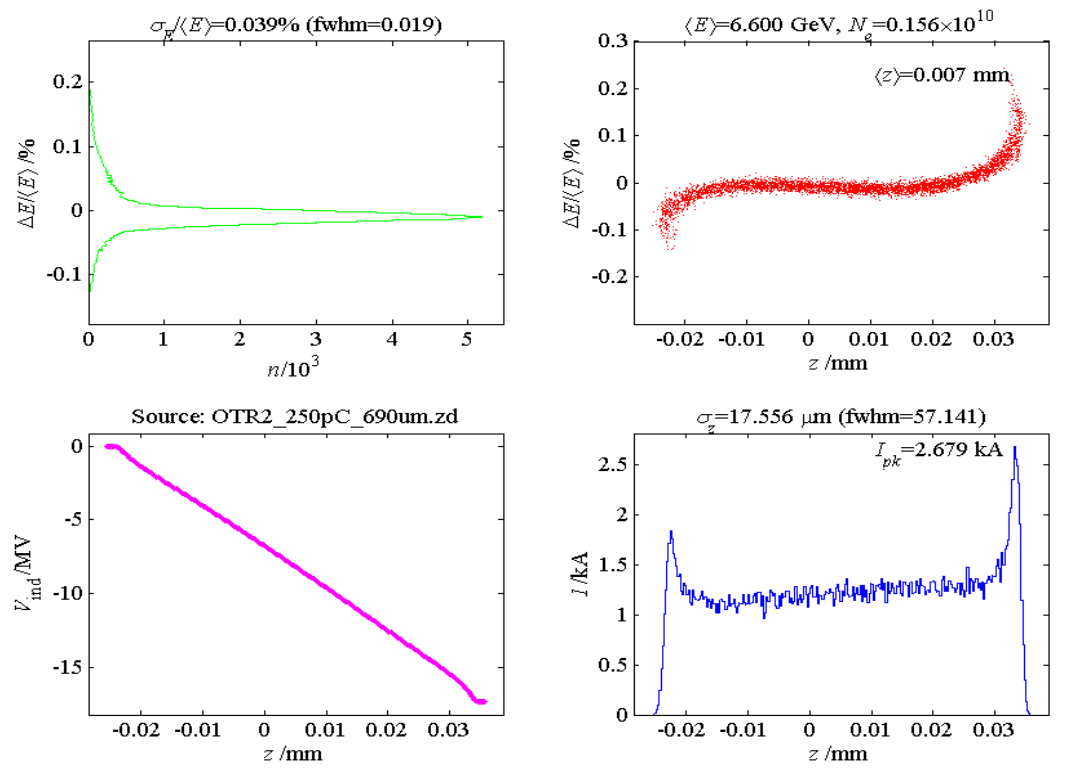

(b)With dechirper $(2 a=1.6 \mathrm{~mm})$.

FIG. 5. LiTrack results of a 250-pC bunch at L3 end in the LCLS (6.6 GeV). The bunch core current is slightly above $1 \mathrm{kA}$. 

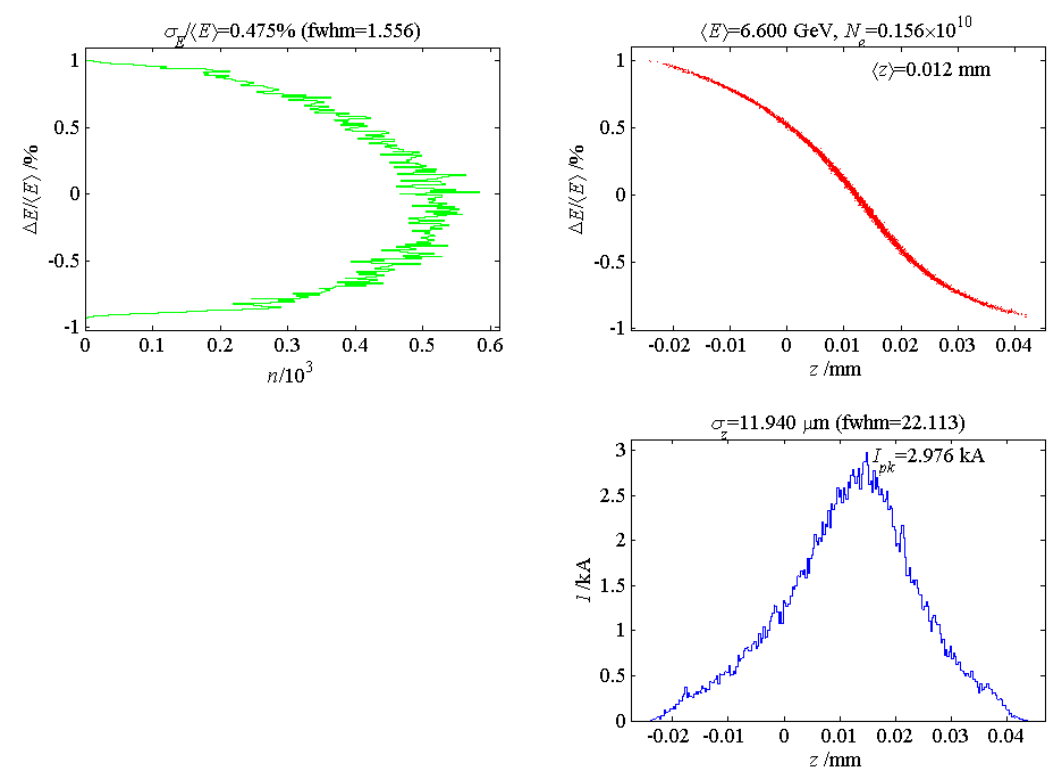

(a)Without dechirper.
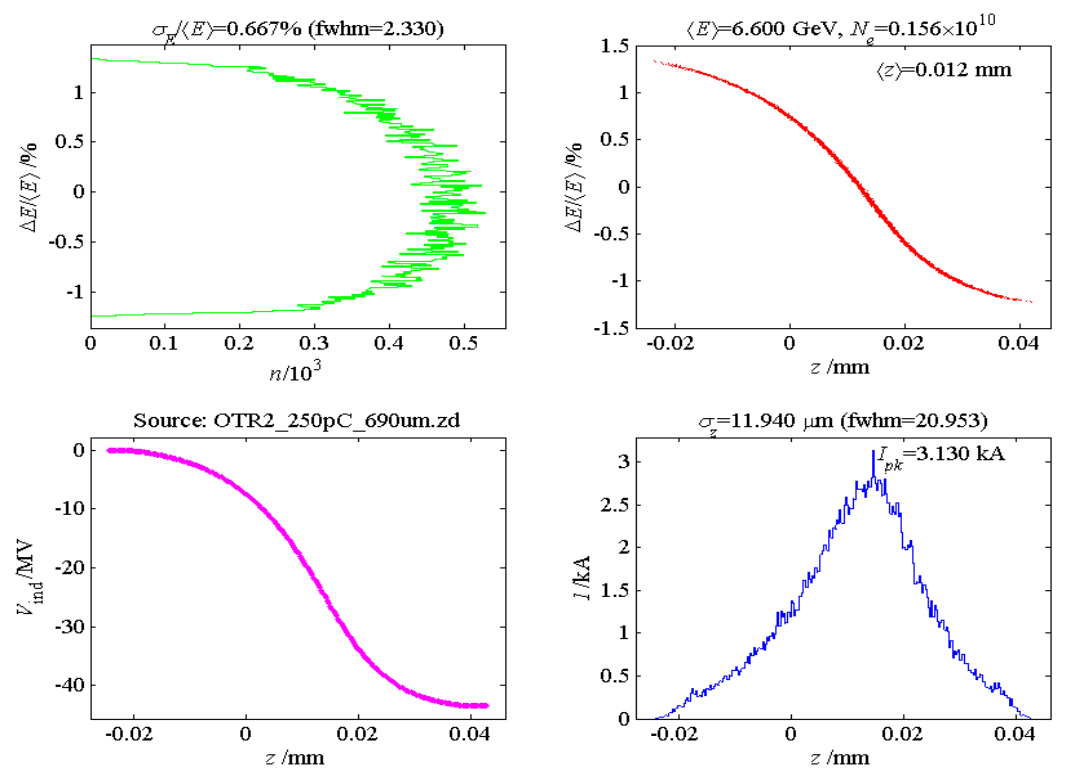

(b)With dechirper $(2 a=1 \mathrm{~mm})$.

FIG. 6. LiTrack results of a $250-\mathrm{pC}$ bunch at L3 end in the LCLS (at 6.6 GeV). The bunch is over-compressed to $3 \mathrm{kA}$ peak current in order to generate a large chirp. 

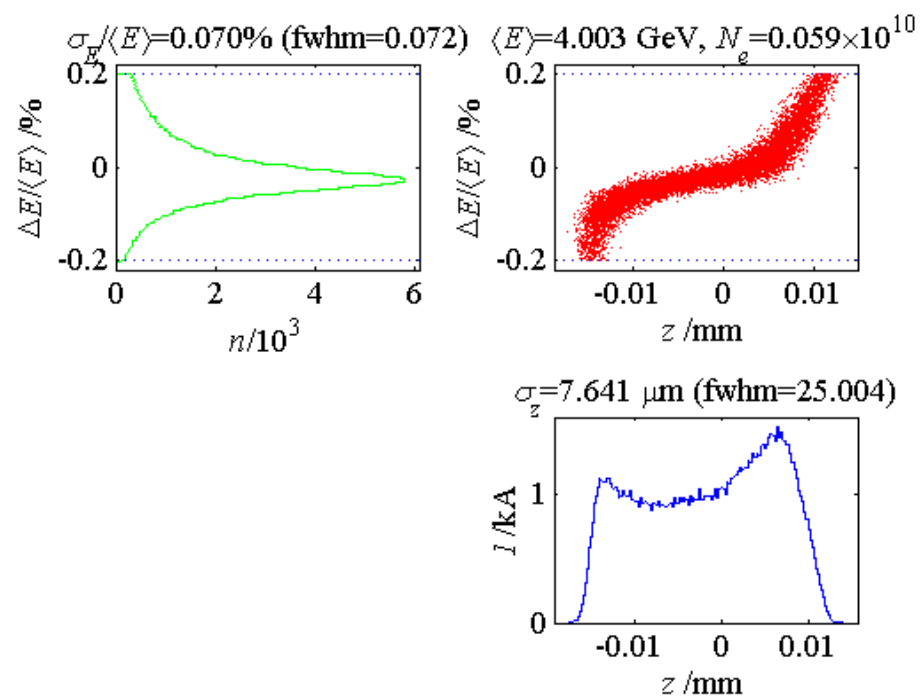

FIG. 7. In LCLS-II after the bypass line, for the nominal (100 pC) electron bunch: longitudinal phase space (upper right), energy spread profile (upper left), and current profile (lower right). This is the result without a dechirper, and we see a significant, residual energy chirp in the bunch.

\section{LCLS-II MOTIVATION}

The LCLS-II accelerator also uses a series of magnetic bunch compressors to generate $1 \mathrm{kA}$ of peak current. With a very large iris, the longitudinal wakefield of the TESLA-type superconducting L-band linac section following the final magnetic compressor is very weak, leaving a large, linear energy chirp in the beam, which will increase the FEL bandwidth significantly. Fortunately, before it reaches the undulator, the beam passes through a $2.2-\mathrm{km}-\mathrm{long}$, stainless steel bypass transport line, where the resistive wall wake removes much of the energy chirp. However, a large chirp still remains, as is shown in Fig. 7 below; here the bunch charge is (the nominal) $100 \mathrm{pC}$, with a peak current of $1 \mathrm{kA}$.

Adding a 10-m long adjustable gap, rectangular dechirper after the linac allows control of the final linear energy chirp as the beam enters the FEL 
undulator. Possible dechirper parameters are $a=1-30 \mathrm{~mm}, p=1 \mathrm{~mm}$, and $t=h=0.5 \mathrm{~mm}$. The final energy chirp after this structure, with $2 a=4 \mathrm{~mm}$, is shown in Fig. 8 (a).

Since LCLS-II can provide high-repetition rate bunches to multiple FEL beamlines (at least two beamlines, SXR and HXR, are in the baseline design), it is desirable to have independent controls of x-ray properties, such as bandwidth and pulse duration, while in the same accelerator configuration. Adding a dechirper prior to the individual FEL beamlines can achieve some of these goals. For example, if the gap is adjusted down to $2 a=2 \mathrm{~mm}$, the final chirp can be increased as shown in Fig. 8 (b); this may satisfy some user requests for large FEL bandwidth (close to $0.6 \% \mathrm{FWHM}$ ). Another potential application is the seeded x-ray pulse length shortening by a chirped electron beam, since only the portion of the bunch within the amplification bandwidth will be amplified in the seeded section. Continuing the example of Fig. 8 (b) for an SXR self-seeded beam, assuming the amplification bandwidth at $1 \mathrm{keV}$ is $1 \times 10^{-3}$ (FWHM), the final x-ray pulse length is estimated to be $\sim 10 \mathrm{fs}$, instead of the 60-fs level obtained when there is no energy chirp [as in Fig. 8 (a)].

\section{CONCLUSION}

We have calculated the strength of the longitudinal and transverse wakefields in a flat dechirper as function of gap, and estimated the resulting induced chirp and effect on projected emittance in a short electron bunch. In addition, through LiTrack simulations, we have demonstrated some ways in which such dechirpers can be used in chirp control in the LCLS and in LCLS-II.

We should note, however, that the corrugation parameters given in Table I for the dechirpers are meant to be suggestive only. The strength of the wakes of the dechirper that are assumed here use analytical approximations. It is known 

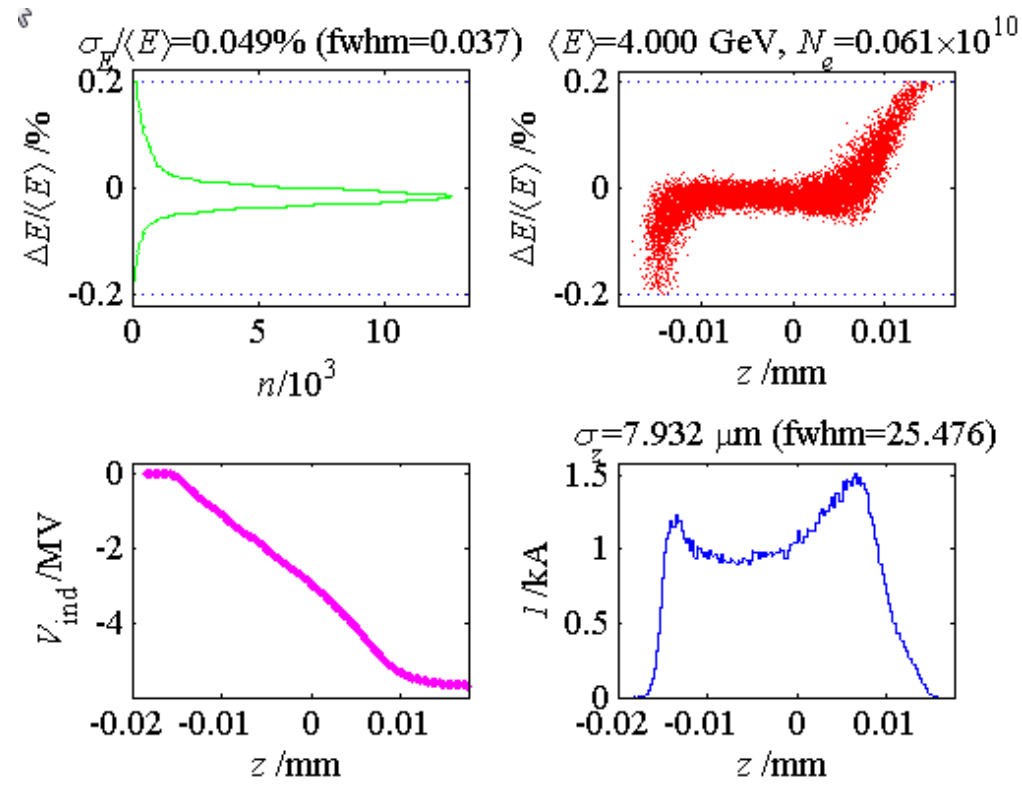

(a)Full gap $2 a=4 \mathrm{~mm}$
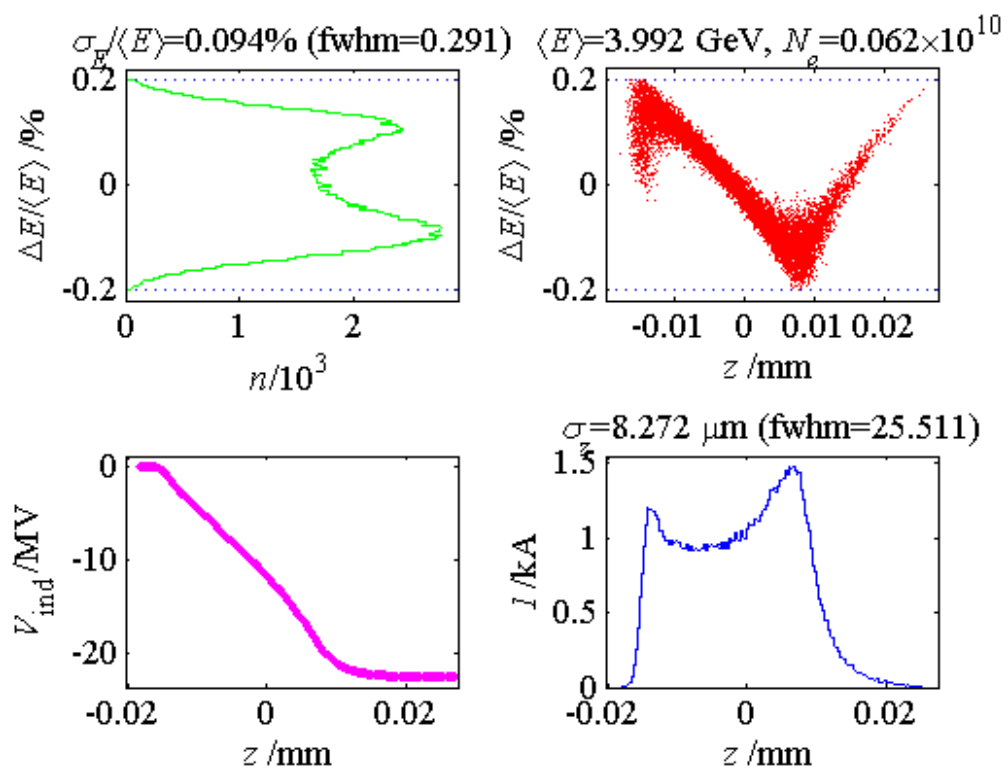

(b)Full gap $2 a=2 \mathrm{~mm}$

FIG. 8. Longitudinal phase space in LCLS-II after the bypass line, and now including a 10-m long dechirper with: (a) full gap $2 a=4 \mathrm{~mm}$, (b) $2 a=2 \mathrm{~mm}$. The bunch charge is the nominal $100 \mathrm{pC}$. The plot at lower left is the wakefield-induced chirp of the 10-m long structure. 
that the real interaction strengths for a given geometry will be somewhat less. Before actually building a dechirper, one needs to calculate the true interaction strength (especially important for the longitudinal wake) by e.g. using a numerical field matching program [5], and then possibly modify the corrugation parameters.

\section{ACKNOWLEDGEMENT}

Work supported by Department of Energy contract DE-AC02-76SF00515.

[1] K. Bane and G. Stupakov, SLAC-PUB-14925 (2012).

[2] P. Emma et al, "Experimental demonstration of energy-chirp control in relativistic electron bunches using a corrugated pipe," submitted to PRL, December 2013.

[3] M. Harrison et al, "Removal of Residual Chirp in Compressed Beams Using a Passive Wakefield Technique," presented at NaPAC13, Pasedena, CA, September 2013, paper MOPHO25.

[4] K. Bane and G. Stupakov, SLAC-PUB-15852 and LCLS-II TN-13-01 (2013).

[5] K. Bane and G. Stupakov, Phys. Rev. ST Accel. Beams 6 (2003) 023301.

[6] K. Bane and P. Emma, Proc. 2005 Part. Accel. Conf., Knoxville, TN, 2005, p. 4266.

[7] S. Serkez et al, DESY 13-109, 2013.

[8] J.Welch et al, Proc. FEL2011, Shanghai, China, 2011, p. 461. 